\title{
Tables of Transport Integrals: A Supplement
}

\author{
William M. Rogers, ${ }^{1}$ William J. Hall, and Robert L. Powell
}

(A pril 23, 1959)

Tables of values of the transport integrals, defined by

$$
J_{n}(x) \equiv \int_{0}^{x} \frac{e^{z} z^{n} d z}{\left(e^{z}-1\right)^{2}},
$$

\begin{abstract}
were previously published in NBS Circular 595 for values of $n$ from 2 through 17 . In this paper, values are given for the integrals where $n$ is 18 and 20 and where $x$ ranges from 0.2 to 50.0 in steps of 0.2 .
\end{abstract}

Soon after the publication of the Tables of Transport Integrals ${ }^{2}$ an unanticipated need arose for tables of the integrals for $n$ equal to 18 and 20 . The integrals with even values of $n$ are useful in the problem of inverting heat capacity results to obtain lattice frequency spectra for solids. ${ }^{3}$ The technique is similar to the method of moments developed by Montroll. ${ }^{4}$ The integrals with odd values of $n$ are not used in this method, and since there was no other need for the integrals with $n$ above 17 , the integrals with $n=19$ were not calculated. The additional

${ }^{1}$ Present address: Headquarters, Strategic Air Command, Offutt Air Force Base, Nebr.

NBS Circ. 595

3 J. A. Morrison, private communication.

4 E. W. Montroll, J. Chem. Phys. 11, 481 (1943). values were calculated on the IBM 650 computer using the already existent programs and auxiliary numbers. The computed values of the integrals are presented in the following tables.

The mathematical formulation, analyses of errors, and tables of auxiliary numbers were discussed in the previous publication. For $x$ below 10, interpolation should be performed by the technique of using an interpolating polynomial in a table of divided differences of the logarithms of both the arguments and the integral values. For $x$ between 0 and 4, a fifthdegree polynomial should be used; between 4 and 10 , a third-degree polynomial. For $x$ above 10, straightforward Lagrangian interpolation should be performed. For $x$ between 10 and 20, a 4-point formula should be used; between 20 and 35, a 3-point; and above 35 , a 2 -point. 
TRANSPORT INTEGRALS: $J_{n}(x) \equiv \int_{0}^{x} \frac{e^{z} z^{n}}{\left(e^{z}-1\right)^{2}} d z ; n=18,20$

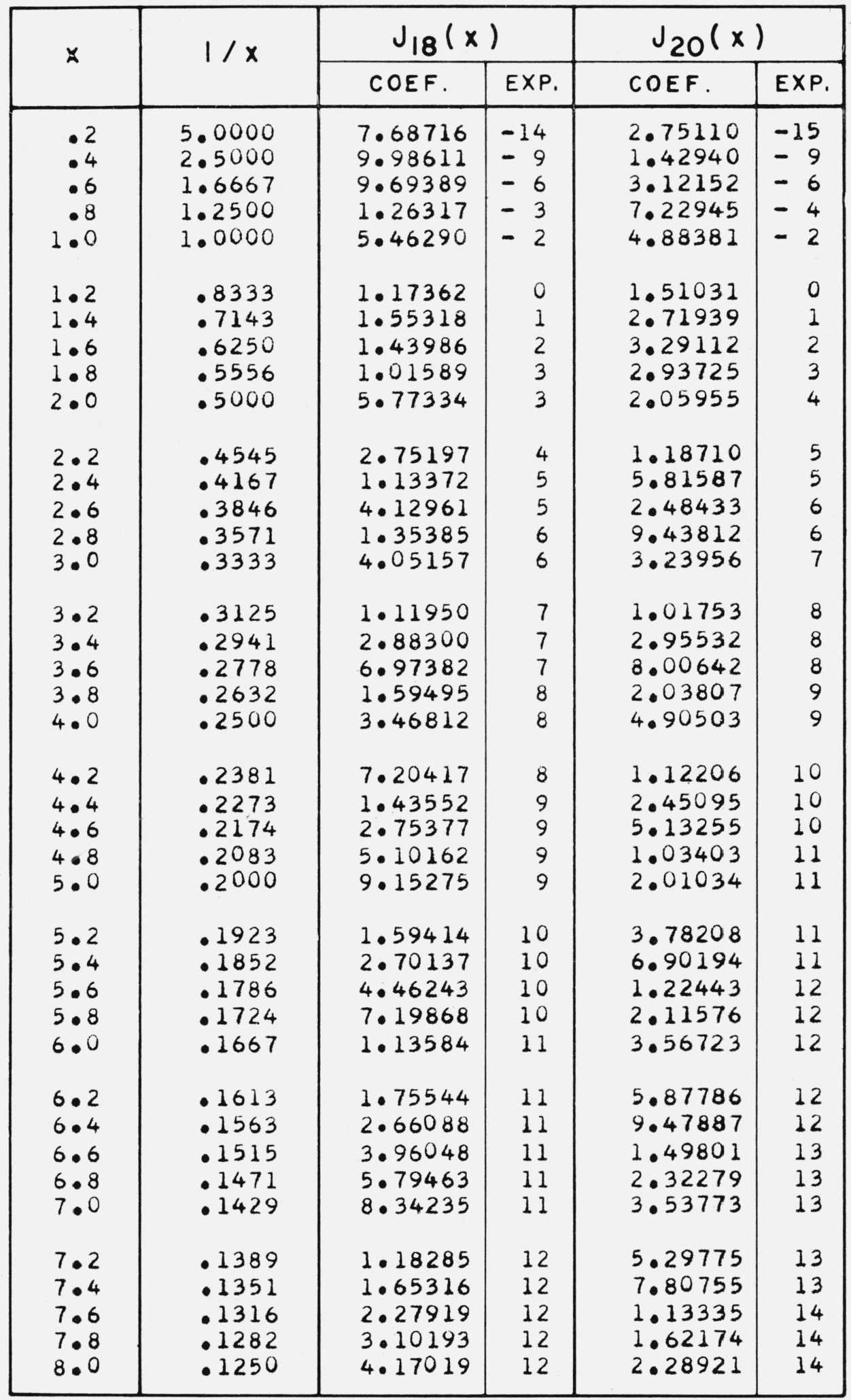


TRANSPORT INTEGRALS: $J_{n}(x) \equiv \int_{0}^{x} \frac{e^{z} z^{n}}{\left(e^{z}-1\right)^{2}} d z ; n=18,20$ (cont'd.)

\begin{tabular}{|c|c|c|c|c|c|}
\hline \multirow[t]{2}{*}{$x$} & \multirow{2}{*}{$1 / x$} & \multicolumn{2}{|c|}{$J_{18}(x)$} & \multicolumn{2}{|c|}{$J_{20}(x)$} \\
\hline & & COEF. & EXP. & COEF & EXP \\
\hline $\begin{array}{l}8.2 \\
8.4 \\
8.6 \\
8.8 \\
9.0\end{array}$ & $\begin{array}{r}1220 \\
.1190 \\
.1163 \\
.1136 \\
.1111\end{array}$ & $\begin{array}{l}5.54145 \\
7.28248 \\
9.47008 \\
1.21916 \\
1.55454\end{array}$ & $\begin{array}{l}12 \\
12 \\
12 \\
13 \\
13\end{array}$ & $\begin{array}{l}3.18984 \\
4.39041 \\
5.97241 \\
8.03411 \\
1.06928\end{array}$ & $\begin{array}{l}14 \\
14 \\
14 \\
14 \\
15\end{array}$ \\
\hline $\begin{array}{r}9.2 \\
9.4 \\
9.6 \\
9.8 \\
10.0\end{array}$ & $\begin{array}{r}.1087 \\
.1064 \\
.1042 \\
.1020 \\
.1000\end{array}$ & $\begin{array}{l}1.96411 \\
2.45996 \\
3.05533 \\
3.76441 \\
4.60253\end{array}$ & $\begin{array}{l}13 \\
13 \\
13 \\
13 \\
13\end{array}$ & $\begin{array}{l}1.40870 \\
1.83786 \\
2.37590 \\
3.04320 \\
3.86510\end{array}$ & $\begin{array}{l}15 \\
15 \\
15 \\
15 \\
15\end{array}$ \\
\hline $\begin{array}{l}10 \cdot 2 \\
10.4\end{array}$ & $\begin{array}{l}.0980 \\
.0962\end{array}$ & $\begin{array}{l}5.58606 \\
6.73205\end{array}$ & $\begin{array}{l}13 \\
13\end{array}$ & $\begin{array}{l}4.86890 \\
6.08530\end{array}$ & $\begin{array}{l}15 \\
15\end{array}$ \\
\hline $10 \cdot 6$ & .0943 & 8.05832 & 13 & 7.54830 & 15 \\
\hline 10.8 & .0926 & 9.58320 & 13 & 9.29500 & 15 \\
\hline 11.0 & .0909 & $1 \cdot 13254$ & 14 & 1.13656 & 16 \\
\hline $11 \cdot 2$ & .0893 & 1.33040 & 14 & 1.38045 & 16 \\
\hline $\begin{array}{l}11.4 \\
11.6\end{array}$ & $\begin{array}{l}.0877 \\
.0862\end{array}$ & $\begin{array}{l}1.55380 \\
1.80461\end{array}$ & $\begin{array}{l}14 \\
14\end{array}$ & $\begin{array}{l}1.66582 \\
1.99760\end{array}$ & $\begin{array}{l}16 \\
16\end{array}$ \\
\hline $\begin{array}{l}11.8 \\
12.0\end{array}$ & $\begin{array}{l}.0847 \\
.0833\end{array}$ & $\begin{array}{l}2.08468 \\
2.39576\end{array}$ & $\begin{array}{l}14 \\
14\end{array}$ & $\begin{array}{l}2.38115 \\
2.82181\end{array}$ & $\begin{array}{l}16 \\
16\end{array}$ \\
\hline $\begin{array}{l}12 \cdot 2 \\
12 \cdot 4\end{array}$ & $\begin{array}{r}.0820 \\
.0806\end{array}$ & $\begin{array}{l}2.73954 \\
3.11761\end{array}$ & $\begin{array}{l}14 \\
14\end{array}$ & $\begin{array}{l}3.32528 \\
3.89742\end{array}$ & $\begin{array}{l}16 \\
16 \\
16\end{array}$ \\
\hline $12 \cdot 6$ & $\begin{array}{r}.0794 \\
0781\end{array}$ & $\begin{array}{l}3.53141 \\
3.98224\end{array}$ & $\begin{array}{l}14 \\
14\end{array}$ & $\begin{array}{l}4.54416 \\
5.27146\end{array}$ & $\begin{array}{l}16 \\
16\end{array}$ \\
\hline 13.0 & .0769 & 4.47120 & 14 & 6.08533 & 16 \\
\hline $\begin{array}{l}13.2 \\
13 \cdot 4 \\
13.6 \\
13 \cdot 8 \\
14.0\end{array}$ & $\begin{array}{r}.0758 \\
.0746 \\
.0735 \\
.0725 \\
.0714\end{array}$ & $\begin{array}{l}4.99927 \\
5.56715 \\
6.17539 \\
6.82427 \\
7.51388\end{array}$ & $\begin{array}{l}14 \\
14 \\
14 \\
14 \\
14\end{array}$ & $\begin{array}{l}6.99174 \\
7.99644 \\
9.10518 \\
1.03233 \\
1.16559\end{array}$ & $\begin{array}{l}16 \\
16 \\
16 \\
17 \\
17\end{array}$ \\
\hline $14 \cdot 2$ & .0704 & $8 \cdot 24402$ & 14 & 1.31077 & 17 \\
\hline $14 \cdot 4$ & .0694 & 9.01431 & 14 & 1.46831 & 17 \\
\hline $14 \cdot 6$ & .0685 & 9.82409 & 14 & 1.63858 & 17 \\
\hline $\begin{array}{l}14.8 \\
15.0\end{array}$ & $\begin{array}{l}.0676 \\
.0667\end{array}$ & $\begin{array}{l}1.06725 \\
1.15583\end{array}$ & $\begin{array}{l}15 \\
15\end{array}$ & $\begin{array}{l}1.82193 \\
2.01862\end{array}$ & $\begin{array}{l}17 \\
17\end{array}$ \\
\hline $\begin{array}{l}15.2 \\
15.4 \\
15.6 \\
15.8 \\
16.0\end{array}$ & $\begin{array}{r}.0658 \\
.0649 \\
.0641 \\
.0633 \\
.0625\end{array}$ & $\begin{array}{l}1.24803 \\
1 \cdot 34370 \\
1.44264 \\
1.54468 \\
1.64960\end{array}$ & $\begin{array}{l}15 \\
15 \\
15 \\
15 \\
15\end{array}$ & $\begin{array}{l}2.22887 \\
2.45283 \\
2.69057 \\
2.94210 \\
3.20737\end{array}$ & $\begin{array}{l}17 \\
17 \\
17 \\
17 \\
17\end{array}$ \\
\hline
\end{tabular}


TRANSPORT INTEGRALS: $J_{n}(x) \equiv \int_{0}^{x} \frac{e^{z} z^{n}}{\left(e^{z}-1\right)^{2}} d z ; n=18,20$ (cont'd.)

\begin{tabular}{|c|c|c|c|c|c|}
\hline \multirow{2}{*}{$x$} & \multirow{2}{*}{$1 / x$} & \multicolumn{2}{|c|}{$J_{18}(x)$} & \multicolumn{2}{|c|}{$J_{20}(x)$} \\
\hline & & COEF & EXP. & COEF. & EXP. \\
\hline $\begin{array}{l}16 \cdot 2 \\
16 \cdot 4 \\
16 \cdot 6 \\
16 \cdot 8 \\
17.0\end{array}$ & $\begin{array}{l}.0617 \\
.0610 \\
.0602 \\
.0595 \\
.0588\end{array}$ & $\begin{array}{l}1.75718 \\
1.86717 \\
1.97933 \\
2.09340 \\
2.20911\end{array}$ & $\begin{array}{l}15 \\
15 \\
15 \\
15 \\
15\end{array}$ & $\begin{array}{l}3.48623 \\
3.77849 \\
4.08386 \\
4.40200 \\
4.73249\end{array}$ & $\begin{array}{l}17 \\
17 \\
17 \\
17 \\
17\end{array}$ \\
\hline $\begin{array}{l}17 \cdot 2 \\
17 \cdot 4 \\
17 \cdot 6 \\
17 \cdot 8 \\
18 \cdot 0\end{array}$ & $\begin{array}{l}.0581 \\
.0575 \\
.0568 \\
.0562 \\
.0556\end{array}$ & $\begin{array}{l}2.32619 \\
2.44436 \\
2.56336 \\
2.68289 \\
2.80270\end{array}$ & $\begin{array}{l}15 \\
15 \\
15 \\
15 \\
15\end{array}$ & $\begin{array}{l}5.07485 \\
5.42855 \\
5.79297 \\
6.16747 \\
6.55134\end{array}$ & $\begin{array}{l}17 \\
17 \\
17 \\
17 \\
17\end{array}$ \\
\hline $\begin{array}{l}18.2 \\
18.4 \\
18.6 \\
18.8 \\
19.0\end{array}$ & $\begin{array}{l}.0549 \\
.0543 \\
.0538 \\
.0532 \\
.0526\end{array}$ & $\begin{array}{l}2.92250 \\
3.04204 \\
3.16107 \\
3.27933 \\
3.39658\end{array}$ & $\begin{array}{l}15 \\
15 \\
15 \\
15 \\
15\end{array}$ & $\begin{array}{l}6.94383 \\
7.34417 \\
7.75153 \\
8.16507 \\
8.58393\end{array}$ & $\begin{array}{l}17 \\
17 \\
17 \\
17 \\
17\end{array}$ \\
\hline $\begin{array}{l}19.2 \\
19.4 \\
19.6 \\
19.8 \\
20.0\end{array}$ & $\begin{array}{l}.0521 \\
.0515 \\
.0510 \\
.0505 \\
.0500\end{array}$ & $\begin{array}{l}3.51262 \\
3.62720 \\
3.74015 \\
3.85127 \\
3.96039\end{array}$ & $\begin{array}{l}15 \\
15 \\
15 \\
15 \\
15\end{array}$ & $\begin{array}{l}9.00721 \\
9.43404 \\
9.86352 \\
1.02948 \\
1.07269\end{array}$ & $\begin{array}{l}17 \\
17 \\
17 \\
18 \\
18\end{array}$ \\
\hline $\begin{array}{l}20 \cdot 2 \\
20 \cdot 4 \\
20 \cdot 6 \\
20 \cdot 8 \\
21 \cdot 0\end{array}$ & $\begin{array}{l}.0495 \\
.0490 \\
.0485 \\
.0481 \\
.0476\end{array}$ & $\begin{array}{l}4.06735 \\
4.17201 \\
4.27423 \\
4.37390 \\
4.47093\end{array}$ & $\begin{array}{l}15 \\
15 \\
15 \\
15 \\
15\end{array}$ & $\begin{array}{l}1.11590 \\
1.15902 \\
1.20198 \\
1.24469 \\
1.28707\end{array}$ & $\begin{array}{l}18 \\
18 \\
18 \\
18 \\
18\end{array}$ \\
\hline $\begin{array}{l}21 \cdot 2 \\
21 \cdot 4 \\
21 \cdot 6 \\
21 \cdot 8 \\
22 \cdot 0\end{array}$ & $\begin{array}{l}.0472 \\
.0467 \\
.0463 \\
.0459 \\
.0455\end{array}$ & $\begin{array}{l}4.56522 \\
4.65671 \\
4.74534 \\
4.83107 \\
4.91386\end{array}$ & $\begin{array}{l}15 \\
15 \\
15 \\
15 \\
15\end{array}$ & $\begin{array}{l}1.32905 \\
1.37056 \\
1.41152 \\
1.45189 \\
1.49159\end{array}$ & $\begin{array}{l}18 \\
18 \\
18 \\
18 \\
18\end{array}$ \\
\hline $\begin{array}{l}22 \cdot 2 \\
22 \cdot 4 \\
22 \cdot 6 \\
22 \cdot 8 \\
23 \cdot 0\end{array}$ & $\begin{array}{l}.0450 \\
.0446 \\
.0442 \\
.0439 \\
.0435\end{array}$ & $\begin{array}{l}4.99369 \\
5.07056 \\
5.14447 \\
5.21543 \\
5.28346\end{array}$ & $\begin{array}{l}15 \\
15 \\
15 \\
15 \\
15\end{array}$ & $\begin{array}{l}1.53058 \\
1.56881 \\
1.60622 \\
1.64278 \\
1.67846\end{array}$ & $\begin{array}{l}18 \\
18 \\
18 \\
18 \\
18\end{array}$ \\
\hline $\begin{array}{l}23 \cdot 2 \\
23 \cdot 4 \\
23 \cdot 6 \\
23 \cdot 8 \\
24 \cdot 0\end{array}$ & $\begin{array}{l}.0431 \\
.0427 \\
.0424 \\
.0420 \\
.0417\end{array}$ & $\begin{array}{l}5.34860 \\
5.41089 \\
5.47037 \\
5.52709 \\
5.58111\end{array}$ & $\begin{array}{l}15 \\
15 \\
15 \\
15 \\
15\end{array}$ & $\begin{array}{l}1.71322 \\
1.74703 \\
1.77987 \\
1.81173 \\
1.84259\end{array}$ & $\begin{array}{l}18 \\
18 \\
18 \\
18 \\
18\end{array}$ \\
\hline
\end{tabular}


TRANSPORT INTEGRALS: $J_{n}(x) \equiv \int_{0}^{x} \frac{e^{z} z^{n}}{\left(e^{z}-1\right)^{2}} d z ; n=18,20$ (cont'd.)

\begin{tabular}{|c|c|c|c|c|c|}
\hline \multirow[t]{2}{*}{$x$} & \multirow{2}{*}{$1 / x$} & \multicolumn{2}{|c|}{$J_{18}(x)$} & \multicolumn{2}{|c|}{$J_{20}(x)$} \\
\hline & & COEF. & EXP. & COEF. & EXP. \\
\hline $\begin{array}{l}24 \cdot 2 \\
24 \cdot 4 \\
24 \cdot 6 \\
24 \cdot 8 \\
25 \cdot 0\end{array}$ & $\begin{array}{l}.0413 \\
.0410 \\
.0407 \\
.0403 \\
.0400\end{array}$ & $\begin{array}{l}5.63250 \\
5.68132 \\
5.72765 \\
5.77155 \\
5.81312\end{array}$ & $\begin{array}{l}15 \\
15 \\
15 \\
15 \\
15\end{array}$ & $\begin{array}{l}1.87243 \\
1.90126 \\
1.92907 \\
1.95585 \\
1.98162\end{array}$ & $\begin{array}{l}18 \\
18 \\
18 \\
18 \\
18\end{array}$ \\
\hline $\begin{array}{l}25 \cdot 2 \\
25 \cdot 4 \\
25 \cdot 6 \\
25 \cdot 8 \\
26 \cdot 0\end{array}$ & $\begin{array}{l}.0397 \\
.0394 \\
.0391 \\
.0388 \\
.0385\end{array}$ & $\begin{array}{l}5.85242 \\
5.88954 \\
5 \cdot 92455 \\
5.95755 \\
5.98862\end{array}$ & $\begin{array}{l}15 \\
15 \\
15 \\
15 \\
15\end{array}$ & $\begin{array}{l}2.00638 \\
2.03014 \\
2.05290 \\
2.07470 \\
2.09553\end{array}$ & $\begin{array}{l}18 \\
18 \\
18 \\
18 \\
18\end{array}$ \\
\hline $\begin{array}{l}26 \cdot 2 \\
26 \cdot 4 \\
26 \cdot 6 \\
26 \cdot 8 \\
27 \cdot 0\end{array}$ & $\begin{array}{l}.0382 \\
.0379 \\
.0376 \\
.0373 \\
.0370\end{array}$ & $\begin{array}{l}6.01783 \\
6.04526 \\
6.07101 \\
6.09514 \\
6.11774\end{array}$ & $\begin{array}{l}15 \\
15 \\
15 \\
15 \\
15\end{array}$ & $\begin{array}{l}2.11543 \\
2.13441 \\
2.15249 \\
2.16969 \\
2.18604\end{array}$ & $\begin{array}{l}18 \\
18 \\
18 \\
18 \\
18\end{array}$ \\
\hline $\begin{array}{l}27 \cdot 2 \\
27 \cdot 4 \\
27 \cdot 6 \\
27 \cdot 8 \\
28 \cdot 0\end{array}$ & $\begin{array}{l}.0368 \\
.0365 \\
.0362 \\
.0360 \\
.0357\end{array}$ & $\begin{array}{l}6 \cdot 13889 \\
6 \cdot 15865 \\
6 \cdot 17710 . \\
6.19431 \\
6.21034\end{array}$ & $\begin{array}{l}15 \\
15 \\
15 \\
15 \\
15\end{array}$ & $\begin{array}{l}2.20157 \\
2.21629 \\
2.23025 \\
2.24345 \\
2.25593\end{array}$ & $\begin{array}{l}18 \\
18 \\
18 \\
18 \\
18\end{array}$ \\
\hline $\begin{array}{l}28 \cdot 2 \\
28 \cdot 4 \\
28 \cdot 6 \\
28 \cdot 8 \\
29 \cdot 0\end{array}$ & $\begin{array}{l}.0355 \\
.0352 \\
.0350 \\
.0347 \\
.0345\end{array}$ & $\begin{array}{l}6 \cdot 22528 \\
6 \cdot 23917 \\
6 \cdot 25208 \\
6 \cdot 26407 \\
6.27519\end{array}$ & $\begin{array}{l}15 \\
15 \\
15 \\
15 \\
15\end{array}$ & $\begin{array}{l}2.26772 \\
2.27885 \\
2.28933 \\
2.29921 \\
2.30849\end{array}$ & $\begin{array}{l}18 \\
18 \\
18 \\
18 \\
18\end{array}$ \\
\hline $\begin{array}{l}29 \cdot 2 \\
29.4 \\
29 \cdot 6 \\
29 \cdot 8 \\
30.0\end{array}$ & $\begin{array}{l}.0342 \\
.0340 \\
.0338 \\
.0336 \\
.0333\end{array}$ & $\begin{array}{l}6.28550 \\
6.29505 \\
6.30388 \\
6.31205 \\
6.31960\end{array}$ & $\begin{array}{l}15 \\
15 \\
15 \\
15 \\
15\end{array}$ & $\begin{array}{l}2.31722 \\
2.32542 \\
2.33311 \\
2.34031 \\
2.34706\end{array}$ & $\begin{array}{l}18 \\
18 \\
18 \\
18 \\
18\end{array}$ \\
\hline $\begin{array}{l}30.2 \\
30.4 \\
30.6 \\
30.8 \\
31.0\end{array}$ & $\begin{array}{l}.0331 \\
.0329 \\
.0327 \\
.0325 \\
.0323\end{array}$ & $\begin{array}{l}6 \cdot 32657 \\
6 \cdot 33299 \\
6.33892 \\
6.34437 \\
6.34939\end{array}$ & $\begin{array}{l}15 \\
15 \\
15 \\
15 \\
15\end{array}$ & $\begin{array}{l}2.35337 \\
2.35927 \\
2.36478 \\
2.36992 \\
2.37472\end{array}$ & $\begin{array}{l}18 \\
18 \\
18 \\
18 \\
18\end{array}$ \\
\hline $\begin{array}{l}31.2 \\
31.4 \\
31 \cdot 6 \\
31.8 \\
32.0\end{array}$ & $\begin{array}{l}.0321 \\
.0318 \\
.0316 \\
.0314 \\
.0313\end{array}$ & $\begin{array}{l}6 \cdot 35401 \\
6 \cdot 35825 \\
6 \cdot 36214 \\
6 \cdot 36572 \\
6 \cdot 36899\end{array}$ & $\begin{array}{l}15 \\
15 \\
15 \\
15 \\
15\end{array}$ & $\begin{array}{l}2.37918 \\
2.38333 \\
2.38720 \\
2.39079 \\
2.39412\end{array}$ & $\begin{array}{l}18 \\
18 \\
18 \\
18 \\
18\end{array}$ \\
\hline
\end{tabular}


TRANSPORT INTEGRALS: $J_{n}(x) \equiv \int_{0}^{x} \frac{e^{z} z^{n}}{\left(e^{z}-1\right)^{2}} d z ; n=18,20$ (cont'd.)

\begin{tabular}{|c|c|c|c|c|c|}
\hline \multirow[t]{2}{*}{$x$} & \multirow{2}{*}{$1 / x$} & \multicolumn{2}{|c|}{$J_{18}(x)$} & \multicolumn{2}{|c|}{$J_{20}(x)$} \\
\hline & & COEF. & EXP. & COEF. & EXP. \\
\hline $\begin{array}{l}32 \cdot 2 \\
32 \cdot 4 \\
32 \cdot 6 \\
32 \cdot 8 \\
33.0\end{array}$ & $\begin{array}{l}.0311 \\
.0309 \\
.0307 \\
.0305 \\
.0303\end{array}$ & $\begin{array}{l}6 \cdot 37199 \\
6 \cdot 37474 \\
6 \cdot 37726 \\
6 \cdot 37956 \\
6 \cdot 38166\end{array}$ & $\begin{array}{l}15 \\
15 \\
15 \\
15 \\
15\end{array}$ & $\begin{array}{l}2.39722 \\
2.40008 \\
2.40274 \\
2.40520 \\
2.40747\end{array}$ & $\begin{array}{l}18 \\
18 \\
18 \\
18 \\
18\end{array}$ \\
\hline $\begin{array}{l}33 \cdot 2 \\
33.4 \\
33.6 \\
33.8 \\
34.0\end{array}$ & $\begin{array}{l}.030 I \\
.0299 \\
.0298 \\
.0296 \\
.0294\end{array}$ & $\begin{array}{l}6 \cdot 38358 \\
6 \cdot 38533 \\
6 \cdot 38692 \\
6 \cdot 38838 \\
6 \cdot 38970\end{array}$ & $\begin{array}{l}15 \\
15 \\
15 \\
15 \\
15\end{array}$ & $\begin{array}{l}2.40957 \\
2.41151 \\
2.41330 \\
2.41496 \\
2.41648\end{array}$ & $\begin{array}{l}18 \\
18 \\
18 \\
18 \\
18\end{array}$ \\
\hline $\begin{array}{l}34 \cdot 2 \\
34 \cdot 4 \\
34 \cdot 6 \\
34 \cdot 8 \\
35.0\end{array}$ & $\begin{array}{l}.0292 \\
.0291 \\
.0289 \\
.0287 \\
.0286\end{array}$ & $\begin{array}{l}6.39091 \\
6.39200 \\
6.39300 \\
6.39391 \\
6.39473\end{array}$ & $\begin{array}{l}15 \\
15 \\
15 \\
15 \\
15\end{array}$ & $\begin{array}{l}2.41788 \\
2.41917 \\
2.42036 \\
2.42145 \\
2.42245\end{array}$ & $\begin{array}{l}18 \\
18 \\
18 \\
18 \\
18\end{array}$ \\
\hline $\begin{array}{l}35.2 \\
35.4 \\
35.6 \\
35.8 \\
36.0\end{array}$ & $\begin{array}{r}.0284 \\
.0282 \\
.0281 \\
.0279 \\
.0278\end{array}$ & $\begin{array}{l}6.39548 \\
6.39615 \\
6.39677 \\
6.39732 \\
6.39783\end{array}$ & $\begin{array}{l}15 \\
15 \\
15 \\
15 \\
15\end{array}$ & $\begin{array}{l}2.42337 \\
2.42421 \\
2.42499 \\
2.42570 \\
2.42634\end{array}$ & $\begin{array}{l}18 \\
18 \\
18 \\
18 \\
18\end{array}$ \\
\hline $\begin{array}{l}36 \cdot 2 \\
36.4 \\
36.6 \\
36.8 \\
37.0\end{array}$ & $\begin{array}{r}.0276 \\
.0275 \\
.0273 \\
.0272 \\
.0270\end{array}$ & $\begin{array}{l}6.39828 \\
6.39869 \\
6.39907 \\
6.39940 \\
6.39971\end{array}$ & $\begin{array}{l}15 \\
15 \\
15 \\
15 \\
15\end{array}$ & $\begin{array}{l}2.42694 \\
2.42748 \\
2.42798 \\
2.42843 \\
2.42884\end{array}$ & $\begin{array}{l}18 \\
18 \\
18 \\
18 \\
18\end{array}$ \\
\hline $\begin{array}{l}37.2 \\
37.4 \\
37.6 \\
37.8 \\
38.0\end{array}$ & $\begin{array}{r}.0269 \\
.0267 \\
.0266 \\
.0265 \\
.0263\end{array}$ & $\begin{array}{l}6.39998 \\
6.40023 \\
6.40045 \\
6.40065 \\
6.40083\end{array}$ & $\begin{array}{l}15 \\
15 \\
15 \\
15 \\
15\end{array}$ & $\begin{array}{l}2.42922 \\
2.42956 \\
2.42988 \\
2.43016 \\
2.43042\end{array}$ & $\begin{array}{l}18 \\
18 \\
18 \\
18 \\
18\end{array}$ \\
\hline $\begin{array}{l}38 \cdot 2 \\
38.4 \\
38.6 \\
38 \cdot 8 \\
39 \cdot 0\end{array}$ & $\begin{array}{r}.0262 \\
.0260 \\
.0259 \\
.0258 \\
.0256\end{array}$ & $\begin{array}{l}6.40099 \\
6.40114 \\
6.40127 \\
6.40139 \\
6.40150\end{array}$ & $\begin{array}{l}15 \\
15 \\
15 \\
15 \\
15\end{array}$ & $\begin{array}{l}2.43066 \\
2.43087 \\
2.43107 \\
2.43124 \\
2.43140\end{array}$ & $\begin{array}{l}18 \\
18 \\
18 \\
18 \\
18\end{array}$ \\
\hline $\begin{array}{l}39.2 \\
39.4 \\
39.6 \\
39.8 \\
40.0\end{array}$ & $\begin{array}{l}.0255 \\
.0254 \\
.0253 \\
.0251 \\
.0250\end{array}$ & $\begin{array}{l}6.40159 \\
6.40168 \\
6.40175 \\
6.40182 \\
6.40188\end{array}$ & $\begin{array}{l}15 \\
15 \\
15 \\
15 \\
15\end{array}$ & $\begin{array}{l}2.43155 \\
2.43168 \\
2.43180 \\
2.43191 \\
2.43201\end{array}$ & $\begin{array}{l}18 \\
18 \\
18 \\
18 \\
18\end{array}$ \\
\hline
\end{tabular}


TRANSPORT INTEGRALS: $J_{n}(x) \equiv \int_{0}^{x} \frac{e^{z} z^{n}}{\left(e^{z}-1\right)^{2}} d z ; n=18,20$ (cont'd.)

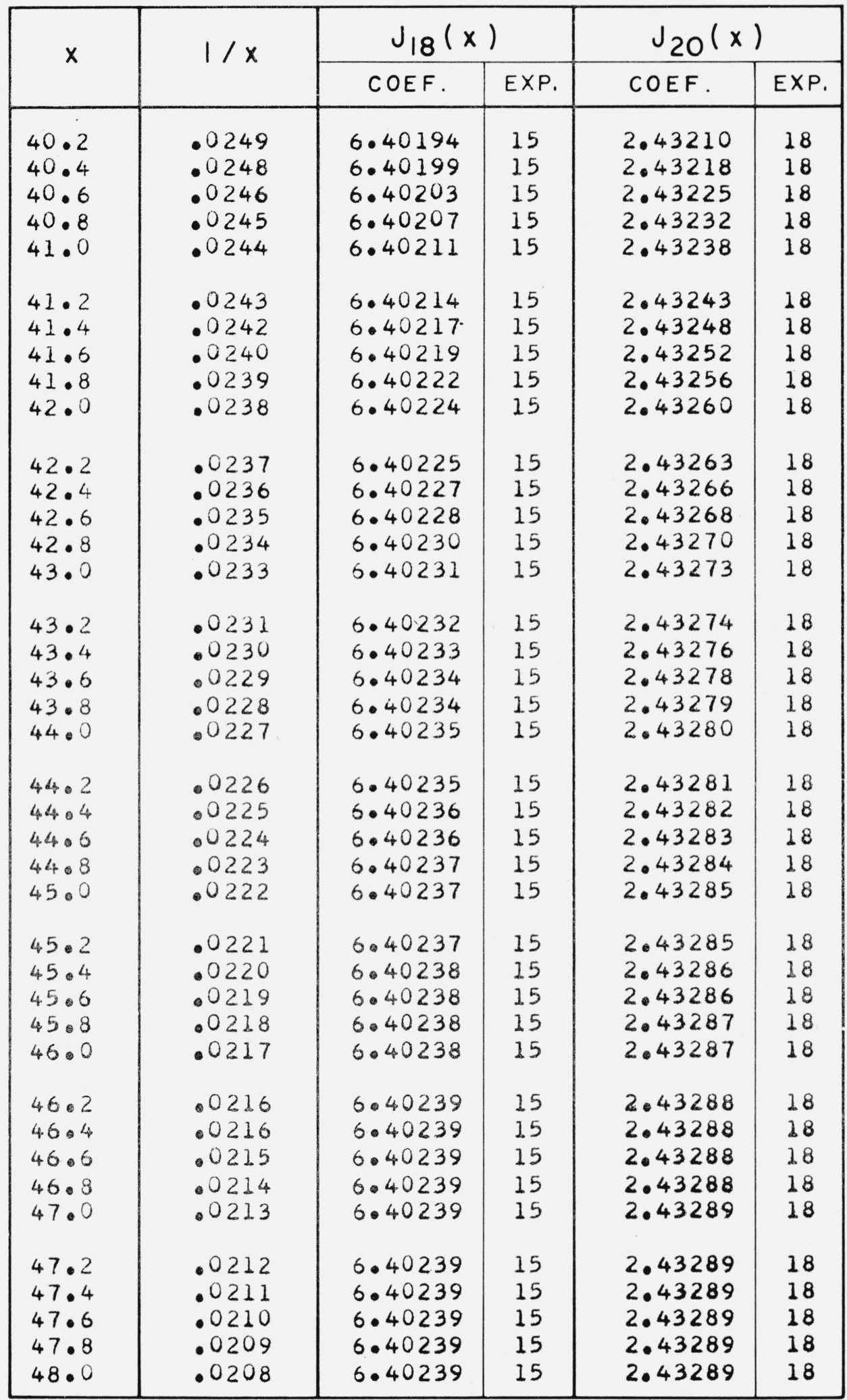


TRANSPORT INTEGRALS: $J_{n}(x) \equiv \int_{0}^{x} \frac{e^{z} z^{n}}{\left(e^{z}-1\right)^{2}} d z ; n=18,20$ (cont'd.)

\begin{tabular}{|c|c|c|c|c|c|}
\hline \multirow{2}{*}{$x$} & $1 / x$ & \multicolumn{2}{|c|}{$J_{18}(x)$} & \multicolumn{2}{c|}{$J_{20}(x)$} \\
\cline { 2 - 6 } & & COEF & EXP. & COEF & EXP. \\
\hline 48.2 & .0207 & 6.40239 & 15 & 2.43290 & 18 \\
48.4 & .0207 & 6.40239 & 15 & 2.43290 & 18 \\
48.6 & .0206 & 6.40240 & 15 & 2.43290 & 18 \\
48.8 & .0205 & 6.40240 & 15 & 2.43290 & 18 \\
49.0 & .0204 & 6.40240 & 15 & 2.43290 & 18 \\
49.2 & .0203 & 6.40240 & 15 & 2.43290 & 18 \\
49.4 & .0202 & 6.40240 & 15 & 2.43290 & 18 \\
49.6 & .0202 & 6.40240 & 15 & 2.43290 & 18 \\
49.8 & .0201 & 6.40240 & 15 & 2.43290 & 18 \\
50.0 & .0200 & 6.40240 & 15 & 2.43290 & 18 \\
\hline
\end{tabular}

Boulder, Colo.

(Paper 63B1-5) 